\title{
CUESTIONARIO DE PROCESO SENSORIAL EN ADULTOS: COMPARACIÓN DE RESULTADOS ENTRE POBLACIÓN DE USA Y DE CHILE: RESULTADOS PRELIMINARES
}

\author{
SENSORY PROCCESING QUESTIONAIRE IN ADULTS: COMPARATION OF RESULTS \\ BETWEEN USA AND CHILE POPULATION: PRELIMINARY RESULTS
}

\section{Silvia Gómez. ${ }^{1}$ Megan Chang, ${ }^{2}$ Dianne Parham, ${ }^{3}$ Erna Imperatore ${ }^{4}$}

\begin{abstract}
Resumen:
Existen escasos estudios sobre la forma de evaluar el proceso sensorial en adultos; una de estas formas es el uso de perfil sensorial desarrollado por Brown y Dunn en el año 2002, aplicable tanto para la población infantil como adulta de habla inglesa. Hasta el momento no existe una evaluación de procesamiento sensorial del adulto validada en castellano. En base a estos antecedentes, el principal objetivo de este estudio es validar la construcción interna de una escala de procesamiento sensorial en el adulto a través del Cuestionario del Proceso Sensorial del Adulto (CPSA) con una población de habla hispana, para luego comparar los resultados con la misma prueba anteriormente valorada en una población de habla inglesa. Los pasos en la elaboración de esta prueba fueron los siguientes: 1) traducción de la prueba al castellano; 2) instalación de la prueba en Internet e invitación a responderla; 3) análisis de los resultados usando estadísticas descriptivas y análisis de factores para observar diferencias con los factores desarrollados con las personas de habla inglesa y 4) comparación entre los factores obtenidos en la prueba con población hispana y con población de habla inglesa.

Se dieron 11 factores que representan hipo o hiper respuesta en los sistemas sensoriales analizados. Estos factores se asemejan a los factores obtenidos con la población de habla inglesa. Esta prueba puede utilizarse para pesquisar dificultades de procesamiento sensorial de adultos en base a los resultados obtenidos
\end{abstract}

\section{Palabras clave:}

procesamiento sensorial, comportamiento, dificultades de desarrollo

\begin{abstract}
:
There are many ways to evaluate sensory processing in children, but less so in adults. One of these evaluations is the Sensory Profile initially developed in children (Dunn, 1999; 2014) and also in adults (Brown \& Dunn, 2002). These tests were developed in English and until this point, there are no assessments of sensory processing validated with Spanish - speaking adults. The main objective of this study is to validate the internal construction of the Adult Sensory Processing Questionnaire Adult Sensory Processing (ASPS) with a Spanish-speaking population, and compare the results with the same test previously validated with an English-speaking (Blanche, Parham, Chang \& Mallinson, 2014). The steps in the development of this test were as follows: 1) translation of original version to Spanish; 2) test upload and invitations to potential responders; 3) analysis of the results using descriptive statistics and factor analysis to evaluate the factor differences between the English and Spanish answers; and 4) comparison between the factors obtained in the test with Spanish and English-speaking population.. The factor analysis yielded 11 factors representing hypo or hyper response in sensory systems. These factors are similar to the factors obtained with the English-speaking population.
\end{abstract} This test may be used in order to screen sensory processing difficulties adult based on the results.

\section{Key words:}

sensory processing, behavior, development difficulties.

1 Terapeuta Ocupacional. Académico Universidad de Chile. 56-2-2978-6587. sgomez@med.uchile.cl

2 Terapeuta Ocupacional. PhD, OTR/L. San José State University. TF 001 (408) 924-3075 Megan.chan@sjsu.edu

3 Terapeuta Ocupacional. PhD, OTR/L FAOTA. University of New Mexico. 001(505)272-8454. DiParham@salud.unm.edu

4 Terapeuta Ocupacional. PhD, OTR/L FAOTA. University of Southern California. 001(323)442-1867. Blanche@chan.usc.edu 


\section{INTRODUCCIÓN}

Procesamiento Sensorial se define como el reconocimiento, la organización y la interpretación de información sensorial (Ayres J. 1972; Miller et al., 2001). Éste influye en el aprendizaje, el comportamiento y elección de actividades (Ayres 1972, 1979; Parham, 1998). Para algunos el Procesamiento Sensorial influye hasta en el estilo de vida relacionado a las relaciones románticas (Jerome \& Liss, 2005). Sus desórdenes se pueden manifestar como hiper sensibilidad o como hipo sensibilidad y se presentan en personas en el espectro del autismo, con dificultades psiquiátricas y en dificultades de aprendizaje y atención (Ahn, Miller, \& Milberger, 2004), (Brown \& Dunn, 2002; Engel-Leyer et al, 2015; Leekman, Nieto, Dunn, 2002).

Para evaluar el proceso sensorial existen muchas pruebas aplicables en niños, pero no en adultos. Una de estas pruebas es el uso de perfil sensorial que fue desarrollado inicialmente en niños (Dunn, 1999) y más tarde en adultos (Brown \& Dunn, 2002; Dunn, 2014). En esta última investigación, las autoras identificaron cuatro patrones de procesamiento sensorial: buscador de sensaciones, evitador de sensaciones, bajo registro e hipersensible. Estos cuestionarios no fueron desarrollados para diferenciar entre los distintos sistemas sensoriales y por lo tanto se enfocan en patrones generalizados de procesamiento sensorial.

Por este motivo en USA se crea el cuestionario de procesamiento sensorial (Adult Sensory Processing Questionnaire - ASPQ), El contenido de este instrumento se basó en la revisión de la literatura y las características específicas de cada sistema. Sus ítems fueron validados por un panel de expertos en el tema (Blanche, Parham, Chang \& Mallinson, 2014).

\section{ANTECEDENTES:}

La relación entre el procesamiento sensorial y el comportamiento se ha estudiado en poblaciones con un diagnóstico establecido. En terapia ocupacional, la mayor parte de la investigación que apoya la relación entre el procesamiento sensorial y comportamiento se ha hecho en niños. Así por ejemplo, Ayres $(1972 ; 1979)$ y Parham (1998) describieron la relación entre las habilidades de aprendizaje y procesamiento sensorial pobre.

También se describe en la literatura la relación entre el procesamiento sensorial anormal en individuos con alteraciones o dificultades de desarrollo. En este ámbito, abundan las auto descripciones del impacto de procesamiento sensorial en personas con autismo (BenSasson, et al, 2009), conociéndose, por ejemplo, que aproximadamente $90 \%$ de individuos en el espectro del autismo y el $5 \%$ de la población general presentan algún tipo de desorden de procesamiento sensorial (Ahn, Miller, et al 2004; Leekman et al, 2006).

La forma en que influye el procesamiento sensorial durante la actividad ha sido poco estudiada. En estudios en psicología, el 'buscador de sensaciones' es descrito como alguien que tiene ese rasgo de la personalidad y que se caracteriza por la participación en deportes vigorosos, búsqueda de aventura u opciones de tipo recreacional que son riesgosas. Según esta línea de investigación, las personas que eligen estas actividades lo hacen para resolver sus necesidades biológicas de experimentar sensaciones intensas lo que le ayuda a mantener un nivel óptimo de alerta (Franques, Auriacombe, Piquemal, Verger, Brisseau-Gimenez, Grabot, y Tignol, 2003; Zuckerman, 1979, 1994). Las escalas que miden este rasgo de la personalidad (búsqueda de sensaciones) no miden necesariamente el procesamiento sensorial, sino que la tendencia a buscar actividades de alto riesgo.

Los patrones de procesamientos sensoriales se han clasificado de diversas maneras. Uno de ellos corresponde al uso del perfil sensorial desarrollado inicialmente en niños (Dunn, 1997). Por otra parte, Brown \& Dunn (2002) han identificado cuatro patrones de procesamiento sensorial del adulto: buscador de sensaciones, evitador de sensaciones, bajo registro, e hipersensible. Ambos cuestionarios tienden a sobre enfocarse en hipersensibilidad al procesamiento sensorial y los ítems no han sido validados por un panel de expertos. El ASPQ incluye ítems que identifican baja y sobre responsividad así como ítems que se centran en un nivel de alerta generalizado. Al incluirse estos últimos, los investigadores podrán examinar la relación entre procesamiento sensorial y nivel de alerta y discriminar entre actividades elegidas que están relacionadas al nivel de alerta y actividades elegidas que están relacionadas a procesamiento sensorial. El ASPQ también ha sido sometido a un análisis de contenido por un grupo de expertos (Blanche et al., 2014).

En este estudio, luego de haber aplicado un cuestionario que captura el procesamiento sensorial de la población de adultos típicos, podremos comprender mejor el procesamiento sensorial de los adultos con alteraciones del desarrollo tales como autismo o défi- 
cit atencional. Entender esta relación ayuda a los terapeutas ocupacionales a recomendar actividades que equilibren las necesidades sensoriales de los usuarios. Además, este estudio vierte luz en la comparación entre las respuestas de la población de habla inglesa y la población de habla hispana.

En base a lo expuesto, el principal objetivo de este estudio es autentificar la validez interna de la versión en español del Adult Sensory Processing Scale (ASPS) o Cuestionario del Proceso Sensorial del Adulto y comparar los resultados con la misma prueba anteriormente desarrollada y usada con una población de habla inglesa (Blanche, Parham, Chang, \& Mallison, 2014).

\section{MÉTODO}

Se usó la versión en español del ASPQ, que contiene 60 ítems. Cabe hacer notar que la versión original del cuestionario (ASPS) contiene 71 ítems y que en el presente estudio se descartaron las preguntas adicionales de relativas al nivel de alerta (arousal), con lo que se aplican los 60 ítems mencionados que, como se muestra en el cuadro $N^{\circ} 1$, fueron agrupados de acuerdo a categorías de procesamiento sensorial.

Cuadro $N^{\circ} 1$ :

Categorías

\begin{tabular}{|c|c|c|}
\hline Sistema & Ítem & Ejemplos de ítems \\
\hline táctil & 14 & $\begin{array}{l}\text { - Me gusta la sensación del barro, greda u } \\
\text { otro material similar. } \\
\text { - Cuando cocino no me gusta ensuciarme } \\
\text { las manos. } \\
\end{array}$ \\
\hline auditivo & 14 & $\begin{array}{l}\text { - Yo no escucho algunos ruidos fuertes. } \\
\text { - Evito situaciones con ruidos fuertes o } \\
\text { inesperados. }\end{array}$ \\
\hline vestibular & 13 & $\begin{array}{l}\text { - Busco actividades con movimientos inten- } \\
\text { sos, como patinar o esquiar. } \\
\text { - Me producen ansiedad los movimientos } \\
\text { bruscos sobre los que o tengo control, } \\
\text { como ser empujado/a. }\end{array}$ \\
\hline propioceptivo & 12 & $\begin{array}{l}\text { - Tiendo a apoyarme físicamente en mue- } \\
\text { bles u otras personas } \\
\text { - Tengo dificultad en graduar la fuerza que } \\
\text { hago sobre los objetos. }\end{array}$ \\
\hline visual & 7 & $\begin{array}{l}\text { - No noto la luz artificial cuando entro a } \\
\text { una habitación. } \\
\text { - Encuentro que necesito más luz que otras } \\
\text { personas cuando trabajo o me alimento. }\end{array}$ \\
\hline TOTAL & 60 & \\
\hline
\end{tabular}


Se contactó, vía Internet, a 500 adultos entre 18 y 65 años de edad. Los participantes expresaron su interés registrándose en el sitio Web correspondiente, en donde la persona aceptó participar (toda la participación fue a través de Internet) y completó la información demográfica requerida que se detalla más adelante y el "Cuestionario de Procesamiento Sensorial" ya mencionado, con la siguiente escala de respuestas que reflejan la importancia de ese ítem:

\section{Cuadro $\mathrm{N}^{\circ}$ 2:}

Escala de respuestas a cada ítem del cuestionario

\begin{tabular}{|c|c|}
\hline Calificación & Importancia \\
\hline A & Mucho \\
\hline B & Sí \\
\hline C & Regular \\
\hline D & Poca \\
\hline E & Nunca \\
\hline
\end{tabular}

Se protegió la identidad de los informantes y se aplicó "Consentimiento Informado" para el manejo de la información y publicación de los resultados obtenidos.

Se recibieron alrededor de 400 respuestas. De ellas se seleccionaron 344 respuestas que eran las que estaban completas en la distribución que se indica. Éstas constituyeron la muestra.

Cuadro $\mathrm{N}^{\circ}$ 3:

Género y edad

\begin{tabular}{|c|c|}
\hline Mujeres entre 18 y 35 años & 166 \\
\hline Mujeres entre 36 y 65 años & 85 \\
\hline Hombres entre 18 y35 años & 56 \\
\hline Hombres entre 36 y 65 años & 37 \\
\hline TOTAL & 344 \\
\hline
\end{tabular}

El tamaño de la muestra se basó en la sugerencia de Comrey y Lee (1992) que dice que se necesita un número mínimo de cinco participantes por variable y existen 60 variables en el cuestionario sensorial. Se usó una escala tipo Likert para las respuestas.

Los datos obtenidos fueron cargados al programa de Statistical Package for the Social Science (SPSS) para el análisis estadístico. Se eligió este programa por haber sido creado para bases de datos de gran tamaño. Para nuestro conocimiento general de la muestra, la información demográfica fue recogida y cifrada para proteger la identidad de los participantes. Esta información incluyó año de nacimiento, género, nivel de educación, y profesión/trabajo/ocupación. El estudio de los datos de la población norteamericana fué revisado por el Comité de Ética de la Universidad del Sur de California y el estudio de la población de habla hispana fue revisado por representantes de la Comisión de Ética del Departamento de Terapia Ocupacional y Ciencia de la Ocupación, de la Facultad de Medicina de la Universidad de Chile.

\section{ANÁLISIS DE DATOS Y RESULTADOS:}

Como se mencionó en el párrafo anterior, el análisis se hizo a través del programa SPSS para el análisis estadístico, se aplicó a cada cuestionario un análisis de factores exploratorio y se calculó la consistencia interna con objeto de analizar la confiabilidad de las preguntas seleccionadas.

De esta manera, para responder al objetivo principal con la información generada, se aplicaron dos análisis: el primero fue el análisis de factores exploratorio en la población chilena, con objeto de descubrir el número de factores que se están midiendo en el cuestionario. Solo aquellos ítem con una carga factorial mayor o igual a 0.40 fueron incluidos en la versión final de cada cuestionario. Este análisis descartó 15 ítems quedando solo 45 ítems en la escala final. El segundo análisis consistió en una comparación entre los factores encontrados en el cuestionario de la versión en inglés con el cuestionario de la versión en castellano. Para esto se estudiaron los ítems que representaban a cada factor en la población chilena y los ítems presentes en la escala de USA.

Los resultados del análisis de factores de la población chilena revelaron 11 factores que representan hipo e hiper respuesta de cada sistema sensorial incluido en el cuestionario. Los 11 factores están descritos en el cuadro número 4 con sus respectivos ítems y con el promedio y la desviación estándar del puntaje final por cada factor. 
Cuadro 4 Factores de la población chilena

\begin{tabular}{|c|c|c|}
\hline & Interpretación de Cada Factor & Promedio (DS) \\
\hline Factor 1 & Hiper responsividad a input vestibular (8 items) & $27.76 \pm 6.90$ \\
\hline Factor 2 & Hiper responsividad a input auditivo (7 ítems) & $20.67 \pm 6.84$ \\
\hline Factor 3 & Hiper responsividad a input visual (4 ítems) & $14.00 \pm 2.80$ \\
\hline Factor 4 & Buscador de input propioceptivo y vestibular (4 ítems) & $12.90 \pm 0.66$ \\
\hline Factor 5 & Hipo responsividad a input propioceptivo y vestibular (7 ítems) & $24.80 \pm 6.20$ \\
\hline Factor 6 & Hiper responsividad a input táctil (4 items) & $13.38 \pm 2.39$ \\
\hline Factor 7 & Buscador de input auditivo (2 ítems) & $6.00 \pm 2.00$ \\
\hline Factor 8 & Hiper responsividad a input táctil social (3 items) & $10.10 \pm 0.78$ \\
\hline Factor 9 & Hipo responsividad a input auditivo (3 ítems) & $11.30 \pm 0.92$ \\
\hline Factor 10 & Hiper actividad (2 items) & $3.30 \pm 0.20$ \\
\hline Factor 11 & Hipo responsividad a táctil (3 ítems) & $10.10 \pm 2.88$ \\
\hline
\end{tabular}

Imperatore Blanche (manuscrito en preparación)

Se descartaron 15 preguntas por su bajo puntaje

El cuadro 5 representa los factores obtenidos de la población norteamericana (Imperatore Blanche et al., 2014) y de la población chilena, la comparación de los factores de la población norteamericana con la población chilena y un ítem que representa el factor correspondiente.

\section{Cuadro 5}

Comparación de factores entre población norteamericana y chilena

\begin{tabular}{|c|c|c|}
\hline $\begin{array}{l}\text { Nombre del Factor en Pobla- } \\
\text { ción Chilena y en la población } \\
\text { Norteamericana }\end{array}$ & $\begin{array}{c}\text { Comparación de los factores entre ambas } \\
\text { poblaciones }\end{array}$ & Ítems Representativos \\
\hline 1. Vestibular (hiper) & $\begin{array}{l}\text { Representado por dos factores: uno repre- } \\
\text { senta inseguridad gravitacional, el otro res- } \\
\text { puesta a input angular. }\end{array}$ & $\begin{array}{l}\text { Me molesta que me muevan hacia atrás en } \\
\text { forma inesperada. }\end{array}$ \\
\hline 2. Auditivo (hiper) & Los mismos ítems con pequeñas variaciones. & $\begin{array}{l}\text { Me molestan los restaurants con ambientes } \\
\text { ruidosos. }\end{array}$ \\
\hline 3. Visual (hiper) & Los mismos ítems con pequeñas variaciones. & $\begin{array}{c}\text { Noto la luz artificial cuando entro en una } \\
\text { habitación. }\end{array}$ \\
\hline $\begin{array}{l}\text { 4. Propioceptivo - vestibular } \\
\text { (buscador) }\end{array}$ & Incluido en un factor vestíbulo propioceptivo. & $\begin{array}{l}\text { Busco actividades que me provean con } \\
\text { experiencias intensas de movimiento. }\end{array}$ \\
\hline $\begin{array}{l}\text { 5.Vestibular - propioceptivo } \\
\text { (hipo) }\end{array}$ & Los mismos ítems. & $\begin{array}{l}\text { Pierdo el equilibrio fácilmente o me siento } \\
\text { inestable cuando camino sobre superficies } \\
\text { inestables (pasto alto, o piedras). }\end{array}$ \\
\hline
\end{tabular}




\begin{tabular}{|c|c|c|}
\hline $\begin{array}{c}\text { Nombre del Factor en Pobla- } \\
\text { ción Chilena y en la población } \\
\text { Norteamericana }\end{array}$ & $\begin{array}{c}\text { Comparación de los factores entre ambas } \\
\text { poblaciones }\end{array}$ & Ítems Representativos \\
\hline 6. Táctil (hiper objetos) & Los mismos ítems con pequeñas variaciones. & $\begin{array}{c}\text { No me gusta la sensación de joyas o ropas } \\
\text { sueltas alrededor de mis muñecas o cintura. }\end{array}$ \\
\hline 7. Auditivo (buscador) & Los mismos ítems con pequeñas variaciones. & $\begin{array}{c}\text { Me gusta asistir a eventos con música fuerte } \\
\text { o subo el volumen cuando estoy solo. }\end{array}$ \\
\hline 8. Táctil (hiper personas) & Los mismos ítems con pequeñas variaciones. & $\begin{array}{c}\text { Evito tumultos o estar muy cerca de otras per- } \\
\text { sonas que me puedan tocar inesperadamente. }\end{array}$ \\
\hline 9. Auditivo (hipo) & Parte de un Factor de hipo respuesta gene- \\
ralizado. & $\begin{array}{c}\text { No escucho ciertos ruidos de fondo (goteos, } \\
\text { personas que se acercan). }\end{array}$ \\
\hline 10. Táctil (hipo) & Los mismos ítems con pequeñas variaciones. & $\begin{array}{c}\text { Me gusta la sensación de barro, greda u otro } \\
\text { material similar en mis manos o cuerpo. }\end{array}$ \\
\hline 11. Hiperactividad & Incluido en el factor vestíbulo-propioceptivo. & Me considero más activo que otras personas. \\
\hline
\end{tabular}

Al comparar los factores obtenidos en la población chilena con los de la población norteamericana, se dieron pocas diferencias y muchas similitudes.

\section{A) Diferencias:}

a. Una diferencia de un factor de hipo respuesta generalizada en el grupo norteamericano que no se dio en el grupo de Chile y que abarcó ítems incluidos en otros factores de hipo respuesta.

b. Otra diferencia fue la existencia de dos factores que describen la hiper respuesta vestibular en el grupo chileno, uno en respuesta a la gravedad y el otro al input vestibular, y sólo uno en el grupo norteamericano.

B) Similitudes:

a. Se observan equivalencias en los ítems que fueron eliminados por no entrar en ningún factor;

b. Similitud entre los factores en ambos países.

El análisis de ambos grupos muestra los siguientes factores:

- hiper respuesta a input vestibular;

- hiper respuesta a input auditivo;

- hiper respuesta a input visual;

- buscador de input propioceptivo y vestibular;

- hypo respuesta a input propioceptivo y vestibular;
- hiper respuesta a input táctil relacionado a objetos;

- hiper respuesta a input táctil relacionado a contacto social;

- buscador de input auditivo;

- hipo respuesta a input auditivo;

- hiper actividad; e hipo respuesta a input táctil (Blanche \& Chang, manuscrito en preparación)

\section{Discusion y CONCLUSIONES}

A través de la bibliografía sobre el tema, es posible observar que las alteraciones en el procesamiento sensorial en los niños son relativamente frecuentes y que una parte importante de esta población las mantiene durante toda su vida. Consideramos que esta última situación puede suceder ya sea por falta de intervención en el momento oportuno o porque debido a su intensidad no se solucionó totalmente el problema. Por otra parte recordemos que, como se expuso al comienzo del documento, existe sólo una investigación sobre el tema (Adult Sensory Proccesing Questionaire, ASPS, publicada por Blanche, Parham, Chang \& Mallinson, 2014) y que fue aplicada a población norteamericana. En este contexto, frecuencia versus evidencia, consideramos que el presente estudio es de gran importancia y que con los resultados obtenidos, cumplimos con el 
propósito de este trabajo, esto es, validar el cuestionario en una población diferente y comparar los resultados.

Estos resultados nos permiten comprobar, por una parte grandes similitudes entre las poblaciones estudiadas, y por otra conocer fundamentadamente el tipo de alteración que afecta a las personas intervenidas. Ambas situaciones facilitarán y permitirán al terapeuta ocupacional, planificar y organizar con mayor exactitud el proceso de intervención requerida. Por otra parte las personas afectadas al conocer el origen de sus dificultades podrán manipular de alguna manera el input, con lo que logrará mejor manejo de situaciones inconfortables.

Si comparamos los resultados obtenidos en la investigación con la población de habla inglesa con los resultados de la población de habla hispana, podemos observar importantes similitudes como ya se expuso, lo que sin duda da sustento a esta investigación.

Así, de acuerdo a lo expresado en este texto, el conocimiento generado de este estudio:

- Proporciona los datos preliminares para el desarrollo del ASPQ en su versión en castellano;

- Proporciona una base para entender el procesamiento sensorial del adulto en poblaciones de habla hispana;

- Provee información sobre el rango típico de procesamiento sensorial;

- Permite analizar la información de ambos grupos en conjunto.

Reiteramos que este es el primer cuestionario sensorial desarrollado con la población de habla hispana, que nos proporciona datos para el desarrollo del ASPQ y que establece una línea de investigación para estudios futuros que identifiquen déficit de procesamiento sensorial en poblaciones con patologías diversas. Finalmente consideramos que su aplicación hará evidente mayor eficacia de la intervención de Terapia Ocupacional en individuos que presentes respuestas sensoriales anormales.

\section{Limitaciones:}

Al ser un estudio preliminar, los resultados que se presentan constituyen el primer eslabón para la validación del cuestionario. Sería necesario replicar este estudio con otras poblaciones de habla hispana, como poblaciones con problemas en salud mental o con diagnóstico en el espectro del autismo. La propuesta anterior permitiría evaluar el valor discriminativo de la prueba y validar su uso en la práctica clínica para así poder recomendar con propiedad diversas estrategias en la selección, discriminación y organización de las ocupaciones de las personas intervenidas. En este mismo contexto, es evidente la necesidad de que los profesionales que intervengan a través del cuestionario, tengan conocimiento y experiencia en integración sensorial.

\section{AGRADECIMIENTOS}

Este estudio no hubiera sido posible sin la colaboración de aproximadamente 400 personas que desinteresadamente se dieron el tiempo y tuvieron la voluntad de responder el cuestionario. A todas esas personas, muchas gracias.

\section{REFERENCIAS BIBLIOGRÁFICAS:}

- Ahn, R., Miller, L.J., Milberger, S., \& McIntosh, D.N. (2004). Prevalence of parents' perceptions of sensory processing disorders among kindergarten children. American Journal of Occupational Therapy, 58(3), 287-302.

- Ayres, A. Jean (1972). Sensory integration and learning disorders. Los Angeles: Western Psychological Corporation.

Ayres, A. J. (1979/2005). Sensory integration and the child: $25^{\text {th }}$ anniversary edition. Los Angeles: Western Psychological Corporation.

Ben- Sasson, et al, (2009) Prevalence and correlates of sensory over responsively from infancy to elementary school.

- Blanche, E. I., Parham, D., Chang, M., \& Mallinson, T. (2014). Development of an Adult Sensory Processing Scale (ASPS). American Journal of Occupational Therapy, 68(5), 531-538.

Brown, C., \& Dunn, W. (2002). Adolescent/adult sensory profile user's manual. San Antonio, TX: Psychological Corporation.

Dunn, W. \& Westman, K. (1997). The sensory profile: The performance of a national sample of children without disabilities. American Journal of Occupational Therapy, 51(1), 25-34. 
Dunn, W (2014). Sensory Profile 2: User's Manual. Psych Corporation. Engel-Yeger, B., MUzio C., Rinosi, G., Solano P., Geoffroy, P. A., Pompili, M., ... \& Serafini, G. (2015). Extreme sensory processing patterns and their relation with clinical conditions among individuals with major affective disorders. Psychiatry research.

Fanchiang, (1996) The other side of the coin. Growing up with a learning disability. AJOT. 50 (8): 647-54.

Franques, Auriacombe, Piquemal, Verger, Brisseau-Gimenez, Grabot, y Tignol, (2003) Sensation seeking as a common factor in opioid dependent subjects. A cross sectional study. Drug and alcohol dependance, $69,121-126$.

Jerome E. M., \& Liss, M. (2005). Relationships between sensory processing style, adult attachment, and coping. Personality and Individual Differences, 38(6), 1341-1352.

Kinnealey, M., \& Fuiek, M. (1999). The relationship between sensory defensiveness, anxiety, depression, and perception of pain in adults. Occupational Therapy International, 6 (3), 195-206.

Kinnealy, M., Koening, K.P., and Smith, S. (2011). Relationships between sensory modulation and social supports and health-related quality of life. American Journal of Occupational Therapy, 65(3), 320-327. doi: 10.5014/ajot.2011.001370.

Kinnealy, M, Oliver, B., and Wilbarger, P. (1995). A phenomenological study of sensory defensiveness in adults. American Journal of Occupational Therapy, 49(5) 444-451. doi: 10.5014/ajot.49.5.444.

Kinnealy M, Oliver B (2002). [Adult Sensory Questionnaire]. Unpublished raw data. Temple University, College of Allied Health Professionals, Department of Occupational Therapy, 3307 North Broad Street, Philadelphia, PA 19140.

Kline, P. (1993). The handbook of psychological testing (2nd Ed.). London: Routledge.

Leekman S. R., Nieto, C., Libby, S. J., Wing, L., \& Gould, J. (2007). Describing the sensory abnormalities of children and adults with autism. Journal of Autism and Developmental Disorders, 37(5), 894-910.

Mehrabian, A. (1995). Theory and evidence bearing on a Scale of Trait Arousability. Current Psychology, 1995, Volume 14, Number 1, Page 3.

Miller et al (2001) An ecological model of sensory modulation: Performance of children with fragile $X$ syndrome autistic disorder attention - deficit/hyperactivity disorder, and sensory modulation dysfunction.

Miller, L. J., \& Lane, S. J. (2000).Toward a consensus in terminology in sensory integration theory and practice: Part 1: Taxonomy of neurophysiological processes. Sensory Integration Special Interest Section Quarterly, 23, 1-4.

Mulligan, 1996). An analysis of score patterns of children with attention disorders on the Sensory Integration and Praxis tests. AJOT. 50 (8): 647-54.
Parham, L. D. (1998). The relationship of sensory integrative development to achievement in elementary students: Four-year longitudinal patterns. Occupational Therapy Journal of Research, 18 (3), 105-127.

Parham, L. D., Ecker, C., Kuhaneck, H. M., Henry, D. A., \& Glennon, T. J. (2007). Sensory Processing Measure. Los Angeles, Western Psychological Services.

Pfeiffer, B., \& Kinnealey, M. (2003). Treatment of sensory defensiveness in adults. Occupational Therapy International, 10 (3), 175-184.

Raine et al ( 1996, 2998) Autonomic nervous system factors underlying, desinhibited, antisocial and violent behavior. Biosocial perspectives and treatment implications. Acad Sci 20, 794, 46-59.

Tavassoli, T., Miller, L.J., Schoen, S. A., Nielsen, D. M., \& Baron-Cohen, S. (2013). Sensory over-responsivity in adults with autism spectrum conditions. Autism, 1362361313477246.

- Zuckerman, 1979, 1994) Behavioral expressions and biosocial bases of sensory seeking. Cambirdge-University Press. 